\title{
DESREPLICAÇÃO DE FLAVONOIDES DE Hymenaea martiana Hayne POR LC-MS/MS
}

\author{
Fernanda Oliveira ${ }^{1 *}$, Anna Paula Sant'Anna da Silva², Amanda Dias de Araújo ${ }^{3}$, Joelma Pessoa Gonçalves ${ }^{2}$, Alexandre \\ Gomes da Silva ${ }^{3}$, Hortênsia Barbosa Pinto ${ }^{4}$, Suzana Vieira Rabêlo ${ }^{5}$, Sarah Raquel Gomes de Lima-Saraiva ${ }^{5}$, Vera Lúcia \\ de Menezes Lima ${ }^{2}$, Márcia Vanusa da Silva ${ }^{2}$, Larissa Araújo Rolim, Jackson Roberto Guedes da Silva Almeida
}

\author{
${ }^{1}$ Pós-graduação em Biotecnologia, UEFS; ${ }^{2}$ Departamento de Bioquímica UFPE, ${ }^{3}$ INSA; ${ }^{4}$ IFSertão-PE; ${ }^{5}$ Núcleo de Estudos e Pesquisas de Plantas \\ Medicinais, UNIVASF; ${ }^{6}$ Central de Análise de Fármacos, Medicamentos e Alimentos, UNIVASF.
}

*fernanda.gso@hotmail.com

\section{INTRODUÇÃO}

0 uso de plantas medicinais está presente por toda a história da humanidade, não apenas para o uso alimentar, como também no uso terapêutico. Durante longo período, as plantas medicinais constituíram o principal recurso terapêutico disponível, mas os avanços tecnológicos trouxeram as drogas sintéticas para o tratamento de enfermidades. Atualmente, a sociedade vem valorizando a utilização de produtos naturais com propriedades terapêuticas, devido à busca por hábitos saudáveis, além de problemas com o tratamento eficiente de certas enfermidades (SOUZA, 2008).

Hymenaea martiana é uma árvore nativa da região Nordeste, conhecida popularmente como "jatobá", utilizada na medicina tradicional para o tratamento de anemia e gastrite (ANSELMO et al., 2012). Vários estudos demonstram a composição química de $H$. martiana, com a presença de alguns flavonoides glicosídicos, como astilbina, eucrifina, engelitina, taxifolina e compostos fenólicos (CARNEIRO et al., 1993; CECHINEL-FILHO et al., 2000; ALMEIDA et al., 2012).

Diante deste contexto, o presente estudo teve como objetivo realizar uma desreplicação para identificar os flavonoides no extrato obtido partir das cascas de Hymenaea martiana.

\section{MATERIAIS E MÉTODOS}

As cascas de $H$. martiana foram coletados na cidade de Petrolina, Pernambuco, Brasil, em julho de 2015, e foram identificados no Herbário da Universidade Federal do Vale do São Francisco (HVASF), com exsicata $n^{\circ} 6444$, coordenadas $09^{\prime \prime} ' 11^{\prime} 04.30^{\circ} \mathrm{S}$, 040'" $18^{\prime} 05.40^{\circ} \mathrm{W}, 357 \mathrm{~m}$ de altura. 0 material foi seco em temperatura média de $40{ }^{\circ} \mathrm{C}$ em estufa de circulação de ar (Ethiktechno ${ }^{\circledR}$, modelo 420 TD), por um período de 72 horas. Após a secagem e completa estabilização, o material foi pulverizado em moinho de facas Quimis ${ }^{\circledR}$.

As cascas foram submetidas à extração no Instituto Nacional do Semiárido (INSA) em Campina Grande-PB, utilizando o equipamento de Extração Acelerada por Solventes (ASE) Thermo Scientific Dionex® ASE 350, equipado com célula extratora de aço inoxidável com fechamento hermético e cartucho de papel $22 \times 50 \mathrm{~mm}$, um frasco de lavagem (rinse) e frascos coletores (vidro transparente) com capacidade de $250 \mathrm{~mL}$. Foi utilizado etanol $99 \%$ como solvente extrator, temperatura de extração $50{ }^{\circ} \mathrm{C}$, tempo estático 15 minutos, fluxo $5 \mathrm{~mL} / \mathrm{min}$, com duas extrações por célula.

Após o processo, a solução extrativa foi concentrada em evaporador Thermo Scientific Rocket Evaporator $\circledast$, na temperatura de $45{ }^{\circ} \mathrm{C}$. O solvente residual foi retirado em estufa de ar circulante Ethiktechno ${ }^{\circledast}$, modelo $420 \mathrm{TD}$, a $45{ }^{\circ} \mathrm{C}$ por 24 horas, obtendo o Extrato Etanólico Bruto (EEB-Hm).

O EEB-Hm foi solubilizado numa mistura $\mathrm{MeOH}: \mathrm{H} 2 \mathrm{O}(3: 7 \mathrm{v} / \mathrm{v})$, que foi submetida a partição líquido-líquido, em funil de separação, sob agitação manual, de forma exaustiva com hexano, clorofórmio e acetato de etila. Após esse procedimento, os solventes foram evaporados em rotaevaporador a uma temperatura média de $50^{\circ}$ $C$, fornecendo a fração hexano $(\mathrm{Hm}-\mathrm{Fr}$. Hx), fração clorofórmio ( $\mathrm{Hm}-\mathrm{Fr} . \mathrm{CHCl}_{3}$ )e fração acetato de etila (Hm-Fr.AcOEt).

A análise em Cromatografia Líquida de Alta Eficiência acoplada ao espectrômetro de massas (LC-MS/MS) foi desenvolvida NPPNSRibeirão Preto, com a fração $\mathrm{Hm}$-Fr.AcOEt, pois foi a fração com melhores resultados no teor de compostos fenólicos totais e flavonoides totais. Esta análise foi realizada utilizando um equipamento de CLAE (Shimadzu Prominence ${ }^{\circledR}$ ), equipada com duas bombas binárias (LC-20AD), unidade de desgaseificação (DGU-20A), amostrador automático (SIL-20AHT), forno (CTO-20A), módulo de comunicação (CBM-20A), detector de arranjo diodo (SPD-M20A) e uma coluna de fase reversa Luna - Phenomenex ${ }^{\oplus}, 250 \mathrm{~mm} \times 4.6$ $\mathrm{mm}, 5 \mu \mathrm{m})$. A fase móvel foi composta por uma mistura de solvente $\mathrm{A}(0,1 \%$ ácido fórmico em água ultrapurificada) e solvente $\mathrm{B}(0,1 \%$ de ácido fórmico em metanol), seguindo um gradiente de concentração, com fluxo de $1 \mathrm{~mL} / \mathrm{min}$.

O cromatógrafo foi acoplado a um espectrômetro de massas Amazon SL ion trap (Bruker Daltonics ${ }^{\circledR}$ ), equipado com ionizador eletrospray e analizador ion trap, nas seguintes condições: capilaridade $3500 \mathrm{~V}$; placa final $500 \mathrm{~V}$; nebulizador $60 \mathrm{psi}$; fluxo de gás 10,0 L.min-1 e temperatura do gás $330^{\circ} \mathrm{C}$.

Os resultados foram analisados utilizando o banco de dados on-line do website GNPS (Global Natural Product Social Molecular Networking) (GNPS, 2017; WANG et al., 2016). Os dados obtidos no cromatógrafo acoplado ao espectrômetro de massas foram convertidos para o formato mzXML diretamente no software DataAnalysis 4.2 (Bruker Daltonics ${ }^{\circledR}$ ), e submetidos à análise de desreplicação no site do GNPS, e as substâncias foram consideradas identificadas na amostra se os espectros de massa obtivessem pelo menos seis íons que combinam e pontuação de cosseno (cosine score) acima de 0,5 (OLIVEIRA et al., 2017).

As fórmulas moleculares e nomenclaturas das substâncias foram obtidas no PubChem (PUBCHEM, 2017) e ChemSpider (CHEMSPIDER, 2017).

\section{RESULTADOS E DISCUSSÃO}

Os estudos de desreplicação são caracterizados pela separação de componentes de matrizes complexas, com a utilização de métodos 
cromatográficos e espectroscópicos, além de estudos de bioatividade. Técnicas hifenadas destacam-se para este tipo de estudo, baseando-se na comparação das informações obtidas com bancos de dados, resultando na detecção de compostos já conhecidos ou até na elucidação estrutural de novos compostos (LANG et al., 2008). Vários compostos naturais já foram identificados utilizando o método de desreplicação, como flavonoides glicosilados (WARIDEL et al., 2001; OLIVEIRA et al., 2017), derivados do ácido cafeico (PAULI et al., 1999), flavonoides e derivados de flavonoides (WOLFENDER et al., 2000) e isoflavonoides (LAMBERT et al., 2005). A Figura 1 apresenta o de í ons totais (TIC) da fração Hm-Fr.AcOEt.

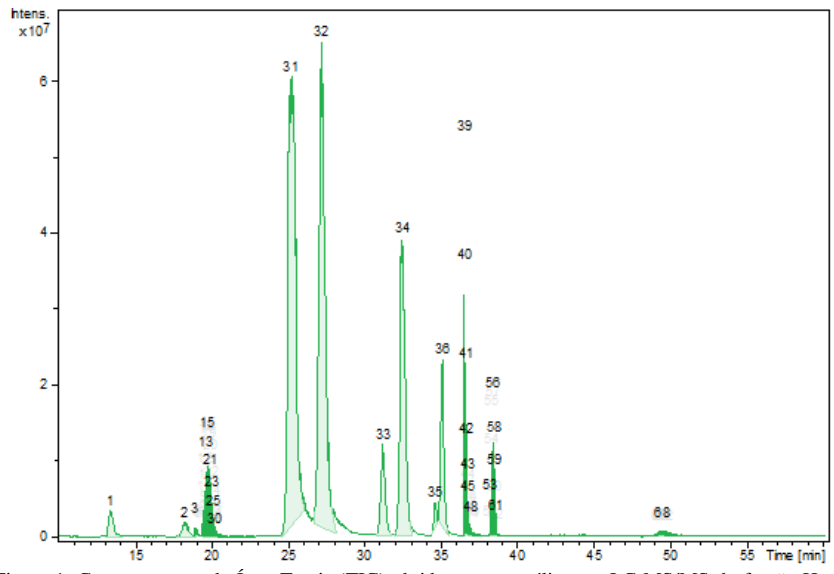

Figura 1. Cromatograma de Íons Totais (TIC) obtido com a análise em LC-MS/MS da fração HmFr.ActOEt (Fonte: Autoria própria).

$\mathrm{Na}$ desreplicação e detecção de flavonoides por LC-MS/MS, utilizando como banco de dados on-line o website GNPS, 18 flavonoides conhecidos foram detectados (Tabela 1), dentre dois flavanonóis, seis flavonóis, quatro isoflavonas, cinco flavonas e um flavan-3-ol.

O flavanonol astilbina foi detectado, flavonoide previamente identificado como o componente majoritário de Hymenaea martiana (CECHINEL et al., 2000). A astilbina é um flavonoide bioativo, com várias atividades farmacológicas já relatadas, como anti-inflamatória (CARNEIRO et al., 1993; LU et al., 2014; DING et al., 2014), anti-isquêmico (DIAO et al., 2014), tratamento de doenç as auto-imunes (GUO et al., 2015), antinociceptivo e antiedematogênico (CECHINEL et al., 2000) e antioxidante (ZHANG et l., 2009; BAKOTA et al., 2015).

Dentre os flavonoides identificados na fração Hm-Fr.AcOEt, apenas a quercetina apresenta atividade fotoprotetora relatada na literatura (STEVANATO et al., 2014; CHOQUENET et al., 2008; EVANS-JOHNSON et al., 2013; VICENTINI et al., 2011). Também apresenta atividade antioxidante (BOOTS et al., 2008; GARDI et al., 2015; RAVICHANDRAN et al., 2014) e atividade anti-inflamató ria relatada (GARDI et al., 2015).

A atividade antioxidante já foi relatada dentre os demais flavonoides identificados, como a taxifolina (CHOBOT et al., 2016), isoquercetina (JIN et al., 2007; VONGSAK et al., 2015), quercetina7-O-ramnosídeo (LIU et al., 2010), kaempferol-7-0-a-ramnosídeo (CHUA et al., 2008), quercitrina (ZHU et al., 2017), ononina (YU et al., 2005), glicitina (GENOVESE et al., 2005), sissotrina (ABDELHADY et al., 2015), amentoflavona (KIM et al., 1998; TARALLO et al., 2011), baicalina (JIN et al., 2007), isovitexina (HE et al., 2016), C-hexosil-apigenina (FERRERES et al., 2009), nobiletina (CHEN et al., 2017). Os flavonoides identificados demonstram o potencial bioativo do extrato das cascas de Hymenaea martiana.

A Tabela 1 apresenta os flavonoides detectados por LC-MS/MS na fração $\mathrm{Hm}-\mathrm{AcOEt}$.
Tabela 1 - Flavonoides detectados por desreplicação (LC-MS/MS) a partir da fração Hm-Fr.AcOEt, obtida a partir do extrato bruto das cascas de Hymenaea martiana.

\begin{tabular}{|c|c|c|c|c|}
\hline $\begin{array}{l}\text { Classe do } \\
\text { flavonoide }\end{array}$ & Metabólito & $\begin{array}{c}\text { Rt } \\
(\min )\end{array}$ & $\begin{array}{l}\text { Fórmula } \\
\text { Molecular }\end{array}$ & $\begin{array}{c}m / z \\
\text { observada } \\
\left(\mathrm{ESI}^{-}\right)\end{array}$ \\
\hline \multirow[t]{2}{*}{ Flavanonol } & Astilbina & 36,5 & $\mathrm{C}_{21} \mathrm{H}_{22} \mathrm{O}_{11}$ & 449 \\
\hline & Taxifolina & 16,1 & $\mathrm{C}_{15} \mathrm{H}_{12} \mathrm{O}_{7}$ & 303 \\
\hline \multirow[t]{6}{*}{ Flavonóis } & Isoquercetina & 36,9 & $\mathrm{C}_{21} \mathrm{H}_{20} \mathrm{O}_{12}$ & 463 \\
\hline & $\begin{array}{l}\text { Quercetina-3-0-alfa- } \\
\text { ramnopiranosídeo }\end{array}$ & 37,8 & $\mathrm{C}_{21} \mathrm{H}_{20} \mathrm{O}_{11}$ & 447 \\
\hline & $\begin{array}{l}\text { Quercetina-7-0-raminosí } \\
\text { deo }\end{array}$ & 42,25 & $\mathrm{C}_{21} \mathrm{H}_{20} \mathrm{O}_{11}$ & 449 \\
\hline & $\begin{array}{l}\text { Kaempferol-7-O-a-L- } \\
\text { ramnosídeo }\end{array}$ & 41,8 & $\mathrm{C}_{21} \mathrm{H}_{20} \mathrm{O}_{10}$ & 431 \\
\hline & Quercetina & 13,0 & $\mathrm{C}_{15} \mathrm{H}_{10} \mathrm{O}_{7}$ & 301 \\
\hline & Quercitrina & 40,4 & $\mathrm{C}_{21} \mathrm{H}_{20} \mathrm{O}_{11}$ & 447 \\
\hline \multirow[t]{4}{*}{ Isoflavonas } & Ononina & 36,3 & $\mathrm{C}_{22} \mathrm{H}_{22} \mathrm{O}_{9}$ & 431 \\
\hline & Glicitina & 41,8 & $\mathrm{C}_{22} \mathrm{H}_{22} \mathrm{O}_{10}$ & 447 \\
\hline & 6"-0-Acetil-Glicitina & 41,9 & $\mathrm{C}_{24} \mathrm{H}_{24} \mathrm{O}_{11}$ & 489 \\
\hline & Sissotrina & 40,5 & $\mathrm{C}_{22} \mathrm{H}_{22} \mathrm{O}_{10}$ & 447 \\
\hline \multirow[t]{5}{*}{ Flavonas } & Amentoflavona & 21,8 & $\mathrm{C}_{30} \mathrm{H}_{18} \mathrm{O}_{10}$ & 539 \\
\hline & Baicalina & 34,3 & $\mathrm{C}_{21} \mathrm{H}_{18} \mathrm{O}_{11}$ & 445 \\
\hline & Isovitexina & 44,1 & $\mathrm{C}_{21} \mathrm{H}_{20} \mathrm{O}_{10}$ & 415 \\
\hline & C-Hexosil-apigenina & 42,1 & $\mathrm{C}_{21} \mathrm{H}_{19} \mathrm{O}_{10}$ & 433 \\
\hline & Nobiletina & 46,2 & $\mathrm{C}_{21} \mathrm{H}_{22} \mathrm{O}_{8}$ & 403 \\
\hline Flavan-3-ol & Isoxantoflavan-3-ol & 11,4 & $\mathrm{C}_{20} \mathrm{H}_{22} \mathrm{O}_{5}$ & 369 \\
\hline
\end{tabular}

Estudos utilizando a estratégia de biomonitoramento que visam o isolamento e purificação para a identificação ou elucidação destes e de flavonoides desconhecidos / não identificados estão sendo desenvolvidos. Métodos cromatográficos analíticos e preparativos (cromatografia em coluna, CLAE e cromatografia em camada delgada) e espectroscópicos (espectrometria de massas e ressonâ ncia magnética nuclear) estão sendo utilizados para tal fim.

\section{CONCLUSÕES}

O estudo fitoquímico do extrato bruto das cascas de Hymenaea martiana demonstra que os compostos fenólicos, principalmente os flavonoides, constituem a classe majoritária da composição quí mica do extrato. Flavonoides foram identificados na espécie pela primeira vez, e os flavonoides astilbina e taxifolina foram identificados no extrato, corroborando estudos anteriores.

O desenvolvimento de estudos mais aprofundados torna-se necessá rio para a identificação dos compostos desconhecidos ou não identificados, visando o estudo fitoquímico aprofundado da espécie em estudo.

\section{REFERÊNCIAS}

ABDELHADY, M.I.S.; KAMAL, A.M.; OTHMAN, S.M.; MUBARAK., M.S.; HADDA, T.B. Total polyphenolic content, antioxidant, cytotoxic, antidiabetic activities, and polyphenolic compounds of Sophora japonica grown in Egypt. Medicinal Chemistry Research, v. 24, p. 482-495, 2015.

ALMEIDA, J.R.G.S.; SILVA, M.E.G.C.; GUIMARÃES, A.L.; OLIVEIRA, A.P.; ARAÚJO, C.S.; SIQUEIRA-FILHO, J.A.; FONTANA, A.P.; DAMASCENO, P.K.F.; BRANCO, C.R.C.; BRANCO, A. HPLC-DAD analysis and antioxidant activity of Hymenaea martiana Hayne (Fabaceae). Journal of Chemical and Pharmaceutical Research, v. 4, n. 2, p. 1160-1166, 2012.

ANSELMO, A.F.; SILVA, C.G.; MARINHO, M.G.V.; ZANELLA, F.C.V.; XAVIER, D.A. Levantamento etnobotânico de plantas medicinais comercializadas por raizeiros em uma feira livre no município de 
Patos - PB. Biofar, Revista de Biologia e Farmácia, v. Especial, p. 39-48, 2012.

BAKOTA, E.L.; WINKLER-MOSER, J.K.; BERHOW, M.A.; PALMQUIST, D.E.; LIU, S.X. Antioxidant activity of hybrid grape pomace extracts derived from midwestern grapes in bulk oil and oil-in-water emulsions. Journal of the American Oil Chemists' Society, vol. 92, p. 1333-1348, 2015.

BOOTS, A.W.; HAENEN, G.R.M.M.; BAST, A. Health effects of quercetin: From antioxidant to nutraceutical. European Journal of Pharmacology, v. 585, p. 325-337, 2008.

CARNEIRO, E.; CALIXTO, J.B.; DELLE MONACHE, F.; YUNES, R.A. Isolation chemical identification and pharmacological evaluation of eucryphin, astilbin and engelitin obtained from the bark of Hymenaea martiana. International Journal of Pharmacognosy, v. 31, n. 1, p. 38-46, 1993.

CECHINEL-FILHO, V.; VAZ, Z.R.; ZUNINO, L.; CALIXTO, J.B.; YUNES, R.A. Antinociceptive and anti-oedematogenic properties of astilbin, taxifolin and some related compounds. Drug Research, v. 50, n. 3, p. E281-E285, 2000.

CHEMSPIDER, Search and share chemistry. Disponível em: < http://www.chemspider.com/>. Acesso em 13/11/2017.

CHEN, X.M.; TAIT, A.R.; KITTS, D.D. Flavonoid composition of orange peel and its association with antioxidant and antiinflammatory activities. Food Chemistry, v. 218, p. 15-21, 2017. CHOBOT, V.; HADACEK, F.; BACHMANN, G.; WECKWERTH, W.; KUBICOVA, L. Pro- and antioxidant activity of three selected flavan type flavonoids: catechin, eriodictyol and taxifolin. International Journal of Molecular Sciences, v. 17, p. 1-9, 2016.

CHOQUENET, B.; COUTEAU, C.; PAPARIS, E.; COIFFARD, L.J.M. Quercetin and rutin as potential sunscreen agents: determination of efficacy by an in vitro method. Journal of Natural Products, v. 71, n. 6, p. 1117-1118, 2008.

CHUA, M.T.; TUNG, Y.T.; CHANG, S.T. Antioxidant activities of ethanolic extracts from the twigs of Cinnamomum osmophloeum. Bioresource Technology, v. 99, p. 1918-1925, 2008.

DIAO, H.; KANG, Z.K.; HAN, F.; JIANG, W. Astilbin protects diabetic rat heart against ischemia-reperfusion injury via blockade of HMGB1-dependent NF-jB signaling pathway. Food and Chemical Toxicology, v. 63, p. 104-110, 2014.

DING, Y.; LIANG, Y.; DENG, B.; QIAO, A.; WU, K.; XIAO, W.; GONG, $\mathrm{W}$. Induction of TGF-b and IL-10 production in dendritic cells using astilbina to inhibit dextran sulfate sodium-induced colitis. Biochemical and Biophysical Research Communications, v. 446, p. 529-534, 2014

EVANS-JOHNSON, J.A.; GARLICK, J.A.; JOHNSON, E.J.; WANGA, X.D.; CHEN, C.Y.O. A pilot study of the photoprotective effect of almond phytochemicals in a 3D human skin equivalent. Journal of Photochemistry and Photobiology B, v. 126, p. 17-25, 2013.

FERRERES, F.; SOUSA, C.; VALENTÃO, P.; ANDRADE, P.B.; SEABRA, R.M.; GIL-IZQUIERDO, A. New C-deoxyhexosyl flavones and antioxidante properties of Passiflora edulis leaf extract. Journal of Agricultural and Food Chemistry, v. 55, p. 10187-10193, 2007. GARDI, C.; BAUEROVA, K.; STRINGA, B.; KUNCIROVA, V.; SLOVAK, L.; PONIST, S.; DRAFI, F.; BEZAKOVA, L.; TEDESCO, I.; ACQUAVIVA, A.; BILOTTO, S.; RUSSO, G.L. Quercetin reduced inflammation and increased antioxidant defense in rat adjuvant arthritis. Archives of Biochemistry and Biophysics, v. 583, p. 150-157, 2015.

GENOVESE, M.I.; HASSIMOTTO, N.M.A.; LAJOLO, F.M. Isoflavone profile and antioxidant activity of Brazilian soybean varieties. Food Science and Technology International, v. 11, n. 3, p.205-211, 2005.

GNPS: Global Natural Products Social Molecular Networking. Disponível em: <https://gnps.ucsd.edu>. Acesso em 13/11/2017. GUO, L.; LIU, W.; LU, T.; GUO, W.; GAO, J.; LUO, Q.; WU, X.; SUN, Y.; WU, X.; SHEN, Y.; XU, Q. Decrease of functional activated T and $B$ cells and treatment of glomerulonephitis in lupus-prone mice using a natural flavonoid astilbina. PLoS ONE, v. 10, n. 4, e0124002, p. 1-15, 2015.

HE, M.; MIN, J.W.; KONG, W.L.; HE, X.H.; LI, J.X.; PENG, B.W. A review on the pharmacological effects of vitexin and isovitexin. Fitoterapia, v. 115, p. 74-85, 2016.

JIN, Y.; LU, Y.; HAN, G.; et al. Comparative study on in vitro antifree radical activities of quercetin, isoquercetin, and rutin. Chinese Traditional Patent Medicine, vol. 38, n. 3, p. 408-412, 2007.

KIM, H.K.; SON, K.H,; CHANG, H.W.; KANG, S.S.; KIM, H.P. Amentoflavone, a plant biflavone: A new potential antiinflammatory agent. Archives of Pharmacal Research, v. 21, n. 4, p. 406-410, 1998.

LAMBERT, M.; STRK, D.; HANSEN, S. H.; SAIRAFIANPOUR, M.; JAROSZEWSKI, J.W. Rapid extract dereplication using HPLC-SPENMR: analysis of isoflavonoids from Smirnowia iranica. Journal of Natural Products, v. 68, p. 1500-1509, 2005.

LANG, G.; MAYHUDIN, N. A.; MITOVA, M. I.; SUN, L.; VAN DER SAR, S.; BLUNT, J. W.; COLE, A. L. J.; ELLIS, G.; LAATSCH, H.; MUNRO, M. H. G. Evolving trends in the dereplication of natural product extracts: new methodology for rapid, small-scale investigation of natural product extracts. Journal of Natural Products, v. 71, p. 1595-1599, 2008.

LIU, H.; MOU, Y.; ZHAO, J.; WANG, J.; ZHOU, L.; WANG, M.; WANG, D.; H. J.; YU, Z.; YANG, F. Flavonoids from Halostachys caspica and their antimicrobial and antioxidant activities. Molecules, vol. 15, p. 7933-7945, 2010.

LU, C.L.; ZHU, W.; WANG, M.; XU, X.J.; LU, C.J. Antioxidant and anti-inflammatory activities of phenolic-enriched extracts of Smilax glabra. Evidence-Based Complementary and Alternative Medicine, v. 2014, 910438, p.1-8, 2014.

OLIVEIRA, G.G.; CARNEVALE-NETO, F.; DEMARQUE, D.P.; PEREIRAJÚNIOR, J.A.S.; PEIXOTO-FILHO, R.C.S.; MELO, S.J.; ALMEIDA, J.R.G.S.; LOPES, J.L.C.; LOPES, N.P. Dereplication of flavonoid glycoconjugates from Adenocalymma imperatoris-maximilianii by untargeted tandem mass spectrometry-based molecular networking. Planta Medica, v. 83, p. 636-646, 2017.

PAULI, G. F.; KUCZKOWIAK, U.; NAHRSTEDT, A. Solvent effects in the structure dereplication of caffeoyl quinic acids. Magnetic Resonance in Chemistry, v. 37, p. 827-836, 1999.

PUBCHEM. Open Chemistry Database. Disponível em: < https://pubchem.ncbi.nlm.nih.gov/>. Acesso em 13/11/2017.

RAVICHANDRAN, R.; RAJENDRAN, M.; DEVAPIRIAM, D. Antioxidant study of quercetin and their metal complex and determination of stability constant by spectrophotometry method. Food Chemistry, v. 146, 472-478, 2014.

SOUZA, A. C. M.; SILVA, M. R. R. Potencial antifúngico de extratos de Hymenaea martiana. Dissertação (Mestrado em Medicina Tropical), Instituto de Patologia Tropical e Saúde Pública, Universidade Federal de Goiás, Goiânia, 2008.

STEVANATO, R.; BERTELLE, M.; FABRIS, S. Photoprotective characteristics of natural antioxidant polyphenols. Regulatory Toxicology and Pharmacology, v. 69, p. 71-77, 2014.

TARALLO, V.; LEPORE, L.; MARCELLINI, M. PIAZ, D.F.; TUDISCO, L.; PONTICELLI, S.; LUND, F.W.; ROEPSTORFF, P.; ORLANDI, A.; PISANO, C.; TOMMASI, N.; FALCO, S. The biflavonoid amentoflavone inhibits neovascularization preventing the activity of proangiogenic vascular endothelial growth factors. Journal of Biological Chemistry, v. 286, v. 22, p. 19641-19651, 2011.

VICENTINI, F.T.M.C.; HE, T.; SHAO, Y.; FONSECA, M.J.V.; VERRI-JR, W.A.; FISHER, G.J.; XU, Y. Quercetin inhibits UV irradiationinduced inflammatory cytokine production in primary human keratinocytes by suppressing NF-kB pathway. Journal of Dermatological Sciences, v. 61, p. 162-168, 2011. 
VONGSAK, B.; MANGMOOL, S.; GRITSANAPAN, W. Antioxidant activity and induction of mRNA expressions of antioxidant enzymes in HEK-293 cells of Moringa oleifera leaf extract. Planta Medica, v. 81, p. 1084-1089, 2015.

WANG, $M$. et al. Sharing and community curation of mass spectrometry data with Global Natural Product Social Molecular Networking. Nature Biotechnology, v. 34, n. 8, p. 828-837, 2016. WARIDEL, P.; WOLFENDER, J-L.; NDJOKO, K.; HOBBY, K. R.; MAJOR, H. J.; HOSTETTMANN, K. Evaluation of quadrupole time-offlight tandem mass spectrometry and ion-trap multiple-stage mass spectrometry for the differentiation of C-glycosidic flavonoid isomers. Journal of Chromatography A, v. 926, p. 29-41, 2001.

WOLFENDER, J-L.; WARIDEL, P.; NDJOKO, K.; HOBBY, K. R.; MAJOR, H. J.; HOSTETTMANN, K. Evaluation of Q-ToF $\neg M S / M S$ and multiple stage IT-MSn for the dereplication of flavonoids and related compounds in crude plant extracts. Analysis, v. 28, p. $895-$ 906, 2000.

YU, D.; DUAN, Y.; BAO, Y.; WEI, C.; AN, L. Isoflavonoids from Astragalus mongholicus protect PC12 cells from toxicity induced by L-glutamate. Journal of Ethnopharmacology, v. 98, p. 89-94, 2005.

ZHANG, Q.F.; ZHANG, Z.R.; CHEUNG, H.Y. Antioxidant activity of rhizoma Smilacis glabrae extracts and its key constituent-astilbin. Food Chemistry, v. 115, p. 297-303, 2009.

ZHU, L.; CHEN, J.; TAN, J.; LIU, X.; WANG, B. Flavonoids from Agrimonia pilosa Ledeb: Free radical scavenging and DNA oxidative damage protection activities and analysis of bioactivity-structure relationship based on molecular and electronic structures. Molecules, v. 22, 195, p. 1-11, 2017. 